NDI MOL2NET, International Conference Series on Multidisciplinary Sciences

http://sciforum.net/conference/mol2net-03

sciforum

\title{
Mathematical modeling of a case of sustainable exploitation of a biological species
}

J. L. González-Santander (E-mail: juanluis.gonzalezsantander@gmail.com) ${ }^{a}$,

G. Martín González (E-mail: german.martin@ucv.es) a .

"Universidad Católica de Valencia "san Vicente mártir"

\begin{tabular}{|l|l|} 
Graphical Abstract & $\begin{array}{l}\text { Abstract } \\
\text { We pose the problem of the introduction of a biological species in an } \\
\text { ecosystem in order to a future exploitation. For the first vivarium } \\
\text { stage, we have obtained a simple method to determine the parameters } \\
\text { of the logistic equation, which determines the growth of the species, } \\
\text { from two experimental measures of the number of individuals. For } \\
\text { the second phase of exploitation, we have obtained an expression for } \\
\text { the maximum exploitation rate (number of individuals caught per } \\
\text { unit time) for it to be sustainable, that is, the population does not } \\
\text { become extinct. In the event that the exploitation rate is higher than } \\
\text { the maximum that ensures sustainability, we provide an expression } \\
\text { for the time of extinction. It has also obtained an expression for the } \\
\text { minimum time that must have the vivarium stage to maximize } \\
\text { sustainable harvest rate. }\end{array}$ \\
\hline
\end{tabular}

\section{Results and discussion}

For the vivarium stage (i.e. $0 \leq t \leq t_{p}$ ), we consider that the population of the species follows the logistic equation.

$$
N(t)=\frac{N_{\infty}}{1-C e^{-k t}},
$$

If $N(0)=N_{0}, N\left(t_{m}\right)=N_{1}$ and $N\left(2 t_{m}\right)=N_{2}$, where $2 t_{m}<t_{p}$, then

$$
\begin{aligned}
& C=\frac{N_{2}\left(N_{0}-N_{1}\right)^{2}}{N_{0}\left(N_{2} N_{0}-N_{1}^{2}\right)}, \\
& k=\frac{1}{t_{m}} \log \left[\frac{N_{2}\left(N_{0}-N_{1}\right)}{N_{0}\left(N_{1}-N_{2}\right)}\right], \\
& N_{\infty}=\frac{N_{1}\left[N_{0}\left(N_{1}-2 N_{2}\right)+N_{2} N_{1}\right]}{N_{1}^{2}-N_{2} N_{0}} .
\end{aligned}
$$

For the exploitation stage, we consider that the population is governed by the following ODE,

$$
\frac{d N}{d \tau}=k N\left(1-\frac{N}{N_{\infty}}\right)-m, \quad N(0)=N_{0}^{*}>0, \quad \tau=t-t_{p} \geq 0,
$$

where $m$ denotes the number of individuals caught per unit time. The solution to the above ODE reads as follows: 
1) Sigmoidal solution $\left(m<k N_{\infty} / 4\right)$

$$
N(\tau)=N_{-}+\frac{N_{+}-N_{-}}{1-K \exp (-\Delta \tau)}
$$

where

$$
\left\{\begin{array}{l}
N_{ \pm}=\frac{1}{2}\left(N_{\infty} \pm \sqrt{N_{\infty}\left(N_{\infty}-4 \frac{m}{k}\right)}\right), \\
\Delta=\sqrt{k\left(k-\frac{4 m}{N_{\infty}}\right)}>0, \quad K=\frac{N_{+}-N_{0}^{*}}{N_{-}-N_{0}^{*}} .
\end{array}\right.
$$

In this case, the species gets extinct when $N_{0}{ }^{*}<N_{\infty} / 2$ or when $m>k N_{0}{ }^{*}\left(1-N_{0}{ }^{*} / N_{\infty}\right)$ at time

$$
\tau_{0}=-\frac{1}{\Delta} \log \left[\frac{\left(N_{0}^{*}-N_{-}\right) N_{+}}{\left(N_{0}^{*}-N_{+}\right) N_{-}}\right] .
$$

2) Trigonometric solution $\left(m>k N_{\infty} / 4\right)$

$$
N(\tau)=\delta-\gamma \tan (\alpha \tau+\beta)
$$

where

$$
\left\{\begin{array}{l}
\alpha=\frac{1}{2} \sqrt{k\left(\frac{4 m}{N_{\infty}}-k\right)}, \quad \tan \beta=\frac{\delta-N_{0}^{*}}{\gamma}, \\
\gamma=\frac{1}{2} \sqrt{\left(\frac{4 m}{k}-N_{\infty}\right) N_{\infty}}, \quad \delta=\frac{1}{2} N_{\infty} .
\end{array}\right.
$$

In this case, the population always gets extinct at time

$$
\tau_{0}=\frac{1}{\alpha}\left[\tan ^{-1}\left(\frac{\delta}{\gamma}\right)-\tan ^{-1}\left(\frac{\delta-N_{0}^{*}}{\gamma}\right)\right] .
$$

3) Singular solution $\left(m=k N_{\infty} / 4\right)$

$$
N(\tau)=\frac{N_{\infty}}{2}+\frac{\left(2 N_{0}^{*}-N_{\infty}\right) N_{\infty}}{k\left(2 N_{0}^{*}-N_{\infty}\right) \tau+2 N_{\infty}} .
$$

In this case, the population gets extinct when $N_{0}{ }^{*}<N_{\infty} / 2$ at time

$$
\tau_{0}=\frac{4 N_{0}^{*}}{k\left(N_{\infty}-2 N_{0}^{*}\right)} \text {. }
$$

According to the above solution, the maximum exploitation rate for which the species does not get extinct is given by

$$
m_{\max }= \begin{cases}k N_{0}^{*}\left(1-\frac{N_{0}^{*}}{N_{\infty}}\right), & N_{0}^{*} \leq \frac{N_{\infty}}{2}, \\ \frac{k N_{\infty}}{4}, & N_{0}^{*}>\frac{N_{\infty}}{2} .\end{cases}
$$

Defining $t_{v}$ as the minimum time we must wait to start the exploitation in order to obtain $m_{\max }$ being sustainable, we have 


$$
t_{v}= \begin{cases}\frac{1}{k} \log \left(\frac{N_{\infty}}{N_{0}}-1\right), & N_{0}<\frac{N_{\infty}}{2}, \\ 0, & N_{0} \geq \frac{N_{\infty}}{2} .\end{cases}
$$

Therefore, that the second stage must start at $t_{p} \geq \max \left(t_{v}, 2 t_{m}\right)$.

\section{References}

(1) Verhulst, Pierre-François (1838), "Notice sur la loi que la population poursuit dans son accroissement", Correspondance mathématique et physique 10: 113-121.

(2) Malthus, Thomas Robert (1798), An Essay on the Principle of Population, Library of Economics, Liberty Fund, Inc., 2000.

(3) Weisstein, Eric W. "Sigmoid Function." From MathWorld--A Wolfram Web Resource. http:// mathworld.wolfram.com/SigmoidFunction.html

(4) Spiegel, Murray R., Manual de fórmulas y tablas matemáticas, McGraw-Hill, 1982.

(5) http://en.wikipedia.org/wiki/Inequality_(mathematics) 